\title{
Identifying the Technical Basis to Represent Long-term Radioactive Waste Form Behavior in Performance Assessments
}

\author{
VICKY FREEDMAN ${ }^{1 *}$, K. PATRICK LEE ${ }^{2}$, DIANA BACON ${ }^{1}$, \\ AND YILIN FANG \\ ${ }^{1}$ Pacfic Northwest National Laboratory, Richland, WA, USA, \\ (*correspondence: vicky.freedman@pnnl.gov) \\ ${ }^{2}$ Orano Federal services ${ }^{2}$
}

Prior to the disposal of hazardous waste in glass and cementitious waste forms, a performance assessment (PA) is conducted to evaluate near-field interactions in the disposal facility and release of contaminant fluxes into the environment. Mathematical models representing waste form performance are often simplified due to computational constraints and lack of a complete understanding of complex processes. Significant conservatisms are used to represent complex interactions among coupled, heterogenous, and transient hydrologic, thermal, and biogeochemical processes over extreme time scales. Confidence in the underlying physical and biogeochemical processes can be significantly enhanced with both field and laboratory testing that quantify these collective processes and assess the conservatism of simpler models. Since demonstration of the long-term safety of waste sites relies on models that predict flow and contaminant transport for thousands of years, this presentation discusses technical gaps and experimental needs associated with informing practical PA models, using the Hanford Site, WA as a case study. These elements support conceptual model refinement of relevant subsystem processes and couplings that represent waste form and sediment interactions under potential disposal conditions. 